AperTO - Archivio Istituzionale Open Access dell'Università di Torino

\title{
Calibration aspects of the JEM-EUSO mission
}

\section{This is a pre print version of the following article:}

Original Citation:

Availability:

This version is available http://hdl.handle.net/2318/1578272

since 2016-06-30T14:52:34Z

Published version:

DOI:10.1007/s10686-015-9453-2

Terms of use:

Open Access

Anyone can freely access the full text of works made available as "Open Access". Works made available under a Creative Commons license can be used according to the terms and conditions of said license. Use of all other works requires consent of the right holder (author or publisher) if not exempted from copyright protection by the applicable law. 


\title{
Calibration Aspects of the JEM-EUSO Mission
}

\author{
The JEM-EUSO Collaboration
}

Received: date / Accepted: date

\begin{abstract}
The JEM-EUSO telescope is, after calibration, a very accurate instrument which yields the number of received photons from the number of measured photo-electrons. Hence, calibration is a crucial part of the instrument and its use. The focal surface (FS) of the JEM-EUSO telescope will consist of about 5000 photo-multiplier tubes (PMTs), which have to be well calibrated to reach the required accuracy in reconstructing the air-shower parameters. The optics system consists of 3 plastic Fresnel (double-sided) lenses of $2.5 \mathrm{~m}$ diameter. The aim of the calibration system is to measure the efficiencies (transmittances) of the optics and absolute efficiencies of the entire focal surface detector. The system consists of 3 main components: (i) Pre-flight calibration devices on ground, where the efficiency and gain of the PMTs will be measured absolutely and also the transmittance of the optics will be. (ii) Onboard relative calibration system applying two methods: a) operating during the day when the JEM-EUSO lid is closed with small light sources on board. b) operating during the night, together with data taking: the monitoring of the
\end{abstract}

\footnotetext{
Contribution to the special issue 'JEM-EUSO' of Experimental Astronomy

JEM-EUSO Collaboration

for full author list see first paper of this volume; corresponding authors:

Philippe Gorodetzky E-mail: philippe.gorodetzky@cern.ch

APC, Univ Paris Diderot, CNRS/IN2P3, CEA/Irfu, Sorbonne Paris Cité, France

Andreas Haungs E-mail: haungs@kit.edu

Karlsruhe Institute of Technology (KIT), IKP, D-76021 Karlsruhe, Germany

Naoto Sakaki E-mail: naoto.sakaki@kit.edu

Karlsruhe Institute of Technology (KIT), IKP, D-76021 Karlsruhe, Germany

Lawrence Wiencke E-mail: lwiencke@mines.edu

Colorado School of Mines, Department of Physics, Golden Colorado, 80401 USA
} 
background rate over identical sites. (iii) Absolute in-flight calibration, again, applying two methods: a) measurement of the moon light, reflected on high altitude, high albedo clouds. b) measurements of calibrated flashes and tracks produced by the Global Light System (GLS). The detail of each calibration method will be described in this paper.

Keywords air-shower fluorescence telescope · JEM-EUSO · calibration · space-based experiment

\section{Introduction}

JEM-EUSO, a telescope looking towards the Earth from the International Space Station (ISS), is dedicated to the detection of photons emitted by extensive air showers (EASs) [1-4]. Secondary particles of these EASs (mainly electrons) excite atmospheric nitrogen causing it to emit fluorescent light. JEMEUSO detects this light in the UV range from the shortest wavelength at which the atmosphere becomes transparent up to the visible range, $290-430 \mathrm{~nm}$. These photons, collected by the optical system of JEM-EUSO, are detected by photomultiplier tubes (PMTs) on the focal surface. The number of fluorescence photons detected by each pixel is summed over 2.5 microsecond intervals called Gate Timing Units (GTUs). The counts per GTU in each pixel are the observables of interest.

The aim of the calibration system is the measure of the efficiencies of the optics and the focal surface detector.

The focal surface of JEM-EUSO, in its present design, will consist of 137 Photo-Detector Modules (PDM) consisting of 9 elementary cells (ECs) each[4]. The ECs are composed of 4 Hamamatsu R11265-M64 multi-anode photomultiplier tubes (MAPMT). An absolute efficiency measurement of the PMTs has to be completed on the ground before the launch because on-board resources for calibration are limited. Only the relative variations of the MAPMT gain and efficiency and the transmittance of the optics will be measured on-board. If the variation of the gains of the MAPMTs as measured is not very large, it can be adjusted by a change of the high-voltage and/or the thresholds in the front-end electronics. Changes in efficiency cannot be modified: the data bases have to be modified accordingly.

The calibration method on the ground (pre-flight) is described in section 2; the foreseen in-flight calibration is summarized in section 3, and section 4 discusses the calibration during operations via moon reflection on clouds and ground-based Global Light System (GLS). 


\section{Pre-flight Ground Calibration}

\subsection{Pre-flight absolute calibration of the Focal Surface}

As the light to be detected is very weak, the PMTs will work in what is called 'single photo-electron' (spe) mode to observe EASs[6]. This is the case because the pulses produced by the front-end discriminators are $25 \mathrm{~ns}$ wide (this will be reduced to $10 \mathrm{~ns}$ in future), the count rate then saturates at approximately $25 \mathrm{MHz}(80 \mathrm{MHz}$ in future). This means that about 30 spe can be present in a GTU (2500 ns) before pile-up deteriorates the efficiency. The maximum photon rate for a $10^{20} \mathrm{eV}$ EAS is about $20 \mathrm{MHz}$.

The EAS reconstruction will take the spe count/GTU per pixel (i.e. one MAPMT anode), and transform it into a Photon/GTU count rate. Then the data from the IR camera and the LIDAR will be used to correct for scattering losses in the atmosphere and clouds effects. Finally, using our knowledge of the fluorescence yield, the number of electrons in the EAS and hence the energy deposited along the EAS track can be found for each GTU. Finally, through hadronic interaction models, this deposited energy is interpreted to obtain the shower's energy[7]. The photon counts per GTU in each pixel and track of the EAS image from pixel to pixel across the focal surface will be used to reconstruct the shower trajectory in the atmosphere[8]. Then with our knowledge of the attitude and ephemeris of the ISS, the arrival direction will be determined.

The first step, conversion of photons striking the PMT photocathode into spe, is the focus of this section. The ratio of the spe count to incident photon count is called the pixel efficiency. This efficiency is the product of the cathode (or quantum) efficiency, which is more or less constant along the PMT surface, and the photo-electron collection efficiency of the tube which varies from pixel to pixel. The photo-cathode is covered by a BG3 filter (290-430 nm). The definition of 'quantum efficiency' used here takes into account the transparency of this filter, which is about $99 \%$ within the relevant bandwidth. The collection efficiency is the percentage of photo-electrons collected by the first dynode and multiplied through the dynode structure to the anode. Thus, electrons are producing a signal that is proportional to the number of photo-electrons emitted by the photo-cathode. The collection efficiency depends on the electrostatic focusing in first stage of the tube, which is not constant in the corners of the photo-cathode in this square MAPMT. This collection efficiency was found to be about $70 \%$ for the Hamamatsu R11265-M64, but varies with the voltage applied between the cathode and first dynode and therefore depends on the gain. So the pixel efficiency varies from pixel to pixel $( \pm 70 \%$ around a central value which is generally around $25 \%$ ).

Fig. 1 shows a typical spectrum of one pixel of a good PMT. The spe charge spectrum shows a large narrow pedestal peak followed by a wider onephotoelectron peak. The gain is defined by the difference between the positions of these two peaks, while the pixel efficiency is proportional to the area of the spe peak. The light source used was faint, producing only one photoelectron 
on average in every 100 gates. This ensures that two photoelectrons/pulses will occur at $1 \%$ of one-spe pulses. The light was generated by a $378 \mathrm{~nm}$ lightemitting diode (LED) inside a $10 \mathrm{~cm}$ diameter integrating sphere, used as a perfect splitter between the output photons (through a diaphragm) and a NIST photodiode used for normalization. The ratio of light between two ports of an integrating sphere is a constant which depends only on the ratio of the port areas. In this way, it is possible to normalize different spectra to the same number of incident photons on a given pixel. The area of the spe peak can be found by integrating above the threshold of a discriminator set at the low point between this peak and the pedestal or by integrating under the peak with the low side extrapolated to zero as shown by a dashed curve. The latter method is representative of the true efficiency, but if we keep the gain unchanged in different runs then the two methods are proportional. In real use, the spe are counted above a threshold by the photon-counting part of the front-end $\mathrm{ASIC}^{1}$.

Fig. 2 shows the efficiencies above threshold versus cathode voltage, together with the efficiencies extrapolated to $-900 \mathrm{~V}$ where the gain is close to $1.0 \times 10^{6}$, the value required by the ASIC used for the front-end electronics of JEM-EUSO. It was observed that the efficiency decreases with high voltage (HV) nearly linearly, i.e. the extrapolation to $-900 \mathrm{~V}$ shows the efficiency is decreased by $13 \%$ from $-1100 \mathrm{~V}$ (the voltage used for sorting the PMTs) to $-900 \mathrm{~V}$ (where they will used in the mission). This is a large factor, explained again by the square geometry of the tubes. The full efficiency does not decrease as fast as the threshold efficiency. The reason (besides the voltage dependence

1 application specific integrated circuit

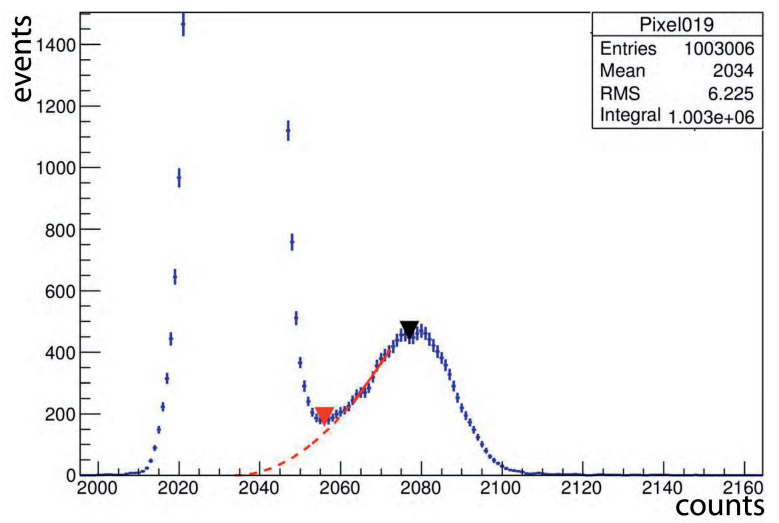

Fig. 1 Single photoelectron (spe) spectrum of one pixel of a good MAPMT. Threshold efficiency corresponds to the spe peak area above the discriminator threshold (red triangle), while the full efficiency corresponds to the area of the spe peak extrapolated to zero (red dashed curve). The horizontal scale is expressed in QDC channels, each channel being $19 \mathrm{fC}$ (CAEN C1205). This spectrum was obtained with the recommended Hamamatsu voltage repartition, with the cathode at $-1100 \mathrm{~V}$. 

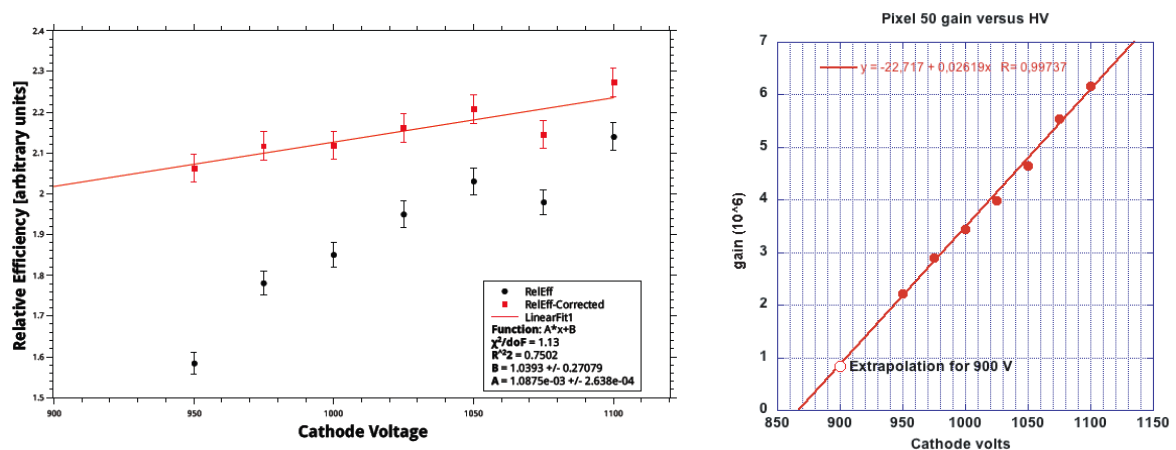

Fig. 2 Left: Relative efficiencies of a pixel for different HV. The black dots show the "threshold efficiencies" and the red ones show the "full efficiencies" (see the text).; Right: gain versus HV.

of the electrostatic focusing effect) is because as the spe peak gets closer to the pedestal, a larger fraction of its area falls below the threshold. Therefore, a measure of the gain and the efficiency is required as a function of the HV applied to the tube.

In JEM-EUSO, the PMTs are grouped into ECs consisting of four $(2 \times 2)$ PMTs. Each EC has its own high voltage power supply (HVPS), so it is necessary to utilize the PMTs with an identical average gain for the chosen HV. We choose a convenient HV, measure the gain of all the pixels of all the PMTs, and then sort them by groups of four [9]. Then, a group of four PMTs are soldered on a socket together with the ASIC containing the front-end electronics for the EC. The PMTs are potted because it is safer in space, especially on the ISS. The efficiency has to be measured for the already potted EC. Also, the anodes are then connected to an ASIC (each ASIC has 64 channels). The circuit for each channel in the ASIC has a variable gain amplifier as its first stage. That makes it possible to set the gains of the 256 pixels in an EC to match. The (photon-counting) discriminator for each channel has its threshold set in the valley between pedestal and spe peak, which is slightly above the minimum between the pedestal and the spe peak. The sensitivity (gain and inverse of the noise) of this ASIC is very high, but the amplifier exhibits non-linearity above two photoelectrons. Therefore the gain of each pixel must be set precisely to $1.0 \times 10^{6}$ in order to avoid distortion of the spe peak. This is reached in a rough way by choosing the right $\mathrm{HV}$ (determined after the sorting the tubes) and by fine tuning with the ASIC amplification.

\subsection{Methods to measure the gains and efficiencies}

The gains are given by the difference in charge channels of the pedestal and the spe peak. Due to this fact they must always be measured in an absolute way using a charge-to-digital converter (QDC). The charge of one QDC channel is 
given by the manufacturer. This does not take into account the integral nonlinearity of the QDC, or other variations. Hence, the QDC must be calibrated by sending a well known current to the input and recording the QDC response as a function of gate of well known width, i.e. the integration time. This gives a measured curve of the QDC response for a known input charge across the full range of the QDC. The input current is given by a stable power supply feeding a resistor, and is measured in series with high accuracy. This allows the estimate of the characteristics of each of the 64 QDC channels to much better than $1 \%$.

The PMT illumination is made from one port of an integrating sphere with the light coming from the right wavelength LED (here $378 \mathrm{~nm}$ ) and one port giving a fraction of the light to a photodiode calibrated at National Institute of Standards and Technology (NIST). For a given distance $(D)$ between the exit of the sphere and the PMT photocathode, a diaphragm of radius $r$ at the sphere exit, the $\cos ^{4} \Theta$-law, arising from a Lambertian light sources, tells us how uniform the illumination is along the PMT. For instance, a diaphragm of $3 \mathrm{~mm}$ diameter and $D=30 \mathrm{~cm}$ will yield a uniform illumination on the $25 \mathrm{~mm}$ of the PMT to an accuracy of $99.6 \%$. The average gains of the PMTs vary from 1.5 to $4.5 \times 10^{6}$ at $-1000 \mathrm{~V}$ and the different pixels exhibit differences of roughly $30 \%$. The adjustable ASIC amplifiers have a range of a factor of four. The gain versus the HV is such that there is a factor of two between -1000 and $-1100 \mathrm{~V}$ and a factor of three between -1000 and $-900 \mathrm{~V}$. What is the minimum acceptable value for the threshold, when expressed in PMT gain? It has to be set above the pedestal base. This corresponds to $2.0 \times 10^{6}$ at $-1100 \mathrm{~V}$, or $1.0 \times 10^{6}$ at $-1000 \mathrm{~V}$. Thus, it is necessary to use $-1100 \mathrm{~V}$ to measure all gains.
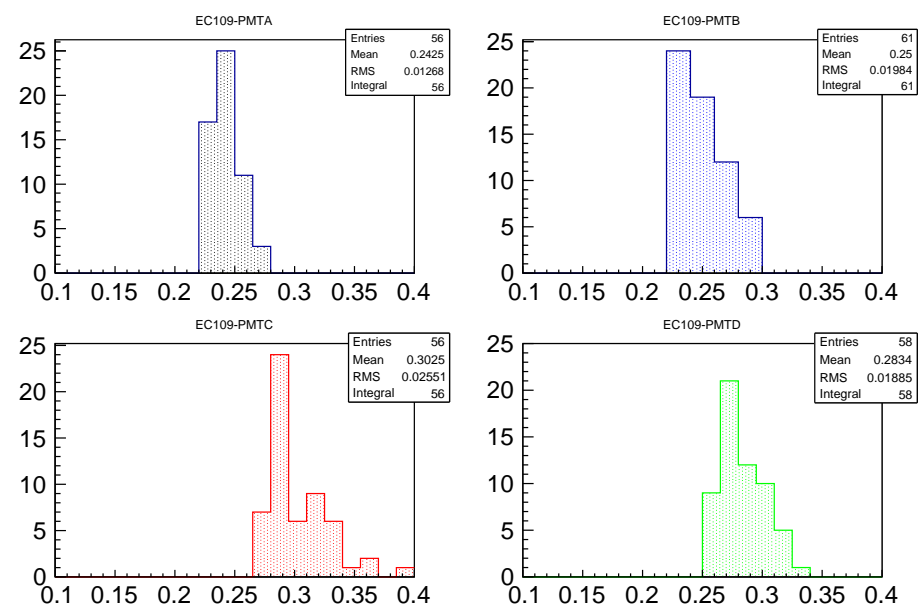

Fig. 3 Histograms of the absolute efficiencies of the 4 PMTs of an EC unit. 
To measure the absolute pixel efficiency, an absolute reference has to be introduced: either a precise light source (difficult to control for reasons linked to the Liouville theorem) or a precise standard light detector, with a surface much larger than the light beam sent to each MAPMT pixel. The latter method was chosen. For the standard light detector, a NIST photodiode (OPHIR, UVPD300), calibrated at all useful wavelengths by NIST to an accuracy of $1.5 \%$ (at one sigma) is used. The light source needs to be very close to the cathode so that the entire photon beam width is fully contained in one pixel. The NIST photodiode on the sphere has a gain close to 1 , while the PMT gain is above $10^{6}$. Hence, the light output to the PMT has to be sent through a collimator made of two holes $\left(1.0\right.$ and $0.3 \mathrm{~mm}$ separated by $20 \mathrm{~mm}$, for a $10^{6}$ reduction). Once the pixels spectra has been recorded, together with the value of the power received by the NIST photodiode, we need to measure the ratio between this power sent to the NIST, and the power sent to the PMT after the collimator. We replace then the PMT by a second NIST photodiode which has also a gain of less than 1, so we have to increase the LED light by a factor of $10^{6}$, enabling an accurate measurement of the ratio between the two NIST diodes. In this way, when measuring only the NIST diode on the sphere, one knows how many photons reach the pixel. The spe spectrum gives the number of corresponding photoelectrons. The ratio between the photoelectrons and the photons defines the efficiency.

In order to aim the narrow beam at a pixel center, one has to place the collimator on the center of a pixel (a square of $2.88 \mathrm{~mm} \times 2.88 \mathrm{~mm}$ ). For that, the integrating sphere is mounted on a remote controlled $\mathrm{X}-\mathrm{Y}$ stage. The spot of light from the sphere will generally touch 4 adjacent pixels. The stage is moved around until the counting rates of these four adjacent pixels are equal. This is done through custom-made software. Then, the sphere is moved half a pixel horizontally and vertically and the light is precisely centered in the center of the pixel. This is extremely precise (within a few microns). For each pixel under calibration, we then examine the signal in the neighboring pixels in order to evaluate the leakage of the electron cascade into the neighboring pixels inside the MAPMT. The real efficiency here is the sum of the signals in the pixel under calibration plus the neighbors. This effect is of the order of 2 $4 \%$, depending on the pixel position on the photocathode. Once two or 3 pixels of the PMT are measured in this absolute way, they become NIST pixels, and, going back to the far illumination (99.6\% uniformity of illumination), we immediately have the absolute efficiencies of the 64 pixels. The PMT efficiencies are approximately $25 \%$, corresponding to a quantum efficiency of $36 \%$, as given by Hamamatsu, and a collection efficiency of about $70 \%$. Fig. 3 shows the histograms of the absolute efficiencies of the 4 PMTs of one EC-unit.

After the absolute efficiencies at $1100 \mathrm{~V}$ are found, the EC units (where 4 PMTs are potted together) are each connected to an ASIC. It is not possible to receive a direct spectrum with this setup because there is no QDC. But it is possible to vary remotely the value of the discriminator threshold with a digital-to-analog converter (DAC) in order to investigate 'S-curves', which are the integrals of the spectra. The derivative of an S-curve yields the spectrum. 
The ASIC has 64 channels, so one run will yield 64 spectra and we adjust the amplifier gains to have identical gains which helps setting an identical discriminator threshold on all pixels. We repeat the same procedure as before: first measure the S-curve with close illumination for the same NIST pixels, and get their efficiencies at $900 \mathrm{~V}$, read with the ASIC, so that we can evaluate the change of efficiency when going from $1100 \mathrm{~V}$ to $900 \mathrm{~V}$, without and with potting. Then we return to the far illumination seen above to get the 64 absolute efficiencies at $900 \mathrm{~V}$. The light sent by the LED is DC, and we can have up to 1.5 pe/GTU before any pile-up appears at the $1 \%$ level. Then a run with 10000 spe (for a $1 \%$ statistical accuracy) in the peak lasts about 20 minutes. This measurement is accurate to better than $3 \%$.

\subsection{Pre-flight measurement of lenses transmittance}

The construction and testing of a 'Bread-Board Model' (BBM) of the JEMEUSO optics was part of the development strategy to mature large area Fresnel lenses to enable space based observations of EAS. The BBM is made of polymethyl-methacrylate (PMMA) as baseline material and uses a flight-like optical prescription appropriate for laboratory testing. The BBM lenses were manufactured in Japan and it was the first experience with the diamond turning machine identified to fabricate the flight lenses. The BBM is $60 \%$ the size of the flight system and represents the center $1.5 \mathrm{~m}$ diameter component of the flight system. Like the flight system, the BBM consists of 3 lenses: two curved double sided Fresnel lenses and a third lens comprised of a Fresnel surface on one side and a diffractive optical element (DOE) on the other (see Fig. 4).
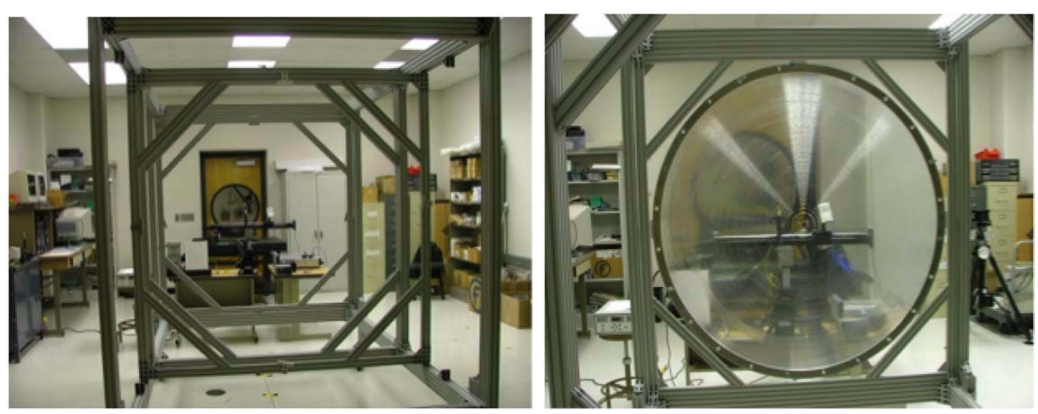

Fig. 4 Lens support frame and metering structure (left) and third BBM lens mounted in frame and metering structure (right). One can see the $60 \mathrm{~cm}$ mirror in the back of the left photo.

The transmittance has been measured at the UAH/CAO laboratory. A 60 $\mathrm{cm}$ diameter parabolic mirror with good reflectance in the near-UV is illuminated by the UV-light source to produce a well-collimated beam that represents a point source at infinity. A source pallet that can use either laser diodes 
or a Xenon lamp with optics was built. The light source is focused onto one end of a silica multi-mode fiber bundle, and the other end is placed at the focal point of the collimator. Filters are introduced to study specific wavelengths of interest to JEM-EUSO. The wavelengths included $405 \mathrm{~nm}, 390 \mathrm{~nm}, 360 \mathrm{~nm}$ and $340 \mathrm{~nm}$. The $405 \mathrm{~nm}$ was produced by a laser diode, and the remaining wavelengths are produced using the Xenon lamp with spike filters with narrow transmission bands, $\approx 5 \mathrm{~nm}$ wide.

The resulting collimated beam was properly characterized and was found to be circular with an azimuthally symmetric Gaussian-shaped intensity profile and a half intensity width of $12.3 \mathrm{~cm}$. The intensity of the collimated beam was measured using a power meter (Newport model 918D-UV-OD3, similar to the NIST photodiodes used in the FS calibration). The transmission of the front lens was measured at normal incidence in 5 positions along a radius and offset from the optical axis: offset $=0$ (optical axis), 10, 20, 30, 40 and $50 \mathrm{~cm}$. These measurements included losses from surface reflection, absorption within the lens material (nominal thickness of $1.5 \mathrm{~cm}$ ), scattering from the Fresnel backcuts and losses due to the surface roughness from the manufacturing of the lenses. The front lens performance was simulated to determine the best focal position to measure the transmitted power. Simulations show the focal length of just the front lens to be $575 \mathrm{~cm}$. The image formed at this position is not a sharp point but is diffuse with the majority $(>90 \%)$ of light concentrated within a spot of area $1 \mathrm{~cm}^{2}$. The transmitted power was measured at several different depths to locate the best focus. The transmitted power was found to be only weakly dependent on the depth of the measurement about the predicted focal depth (Fig. 5). The results of the measured transmission are given in the table 1 .

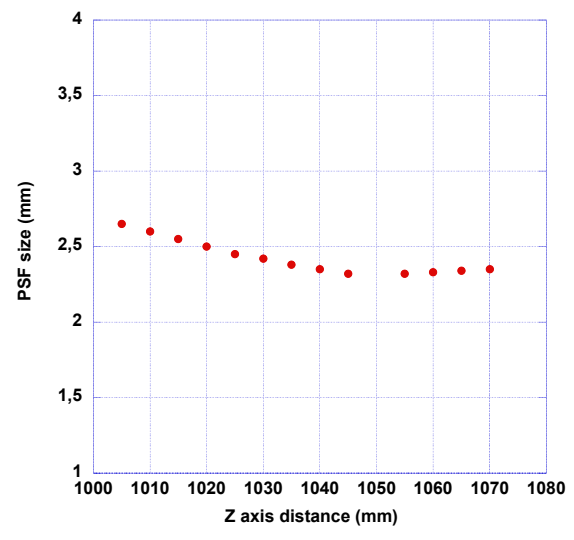

Fig. 5 Image size measured as a function of distance along the optical axis.

The transmitted light is measured with a CCD camera for spot-size measurements and a power meter for the transmission measurements. The camera 
Table 1 Measured transmission data.

\begin{tabular}{ccccl}
\hline $\begin{array}{c}\text { Offset } \\
{[\mathrm{cm}]}\end{array}$ & $\begin{array}{c}\text { Incident } \\
\text { Power }\end{array}$ & $\begin{array}{c}\text { Transmitted } \\
\text { Power }\end{array}$ & $\begin{array}{c}\text { Transmission } \\
{[\%]}\end{array}$ & note \\
\hline 0 & 288 & 171 & 60 & Center hole exists from manufacturing \\
10 & 328 & 189 & 58 & \\
20 & 364 & 207 & 57 & \\
30 & 368 & 254 & 69 & Source begins to illuminate the frame \\
40 & 368 & 231 & 63 & Source is further off the edge of the lens \\
50 & 368 & 158 & 43 & \\
\hline
\end{tabular}

is a Kodak CCD model 1401 e with $1317 \times 1035$ active pixels. Each pixel is square with a dimension of $6.8 \mu \mathrm{m}$. A mechanical shutter is used to control the integration period for each test condition and to insure the signal level is within the dynamic range of the CCD output (12 bit data). The light intensity is measured with the NIST Newport model 818 used to measure the incident flux. This photodiode has a diameter of $1 \mathrm{~cm}$, larger than the point spread function (PSF) of the lens system (which has a diameter $<5 \mathrm{~mm}$ ). The transmission has been measured for on-axis beams, centered on the focal point, for on-axis beams displaced along the lens radius, and then with tilted beams. The measured transmission value of $69 \%$ at $30 \mathrm{~cm}$ offset compares well with the $72 \%$ transmission that was predicted by simulations based on the detailed characterization of the BBM lens and surface roughness, showing that the lenses were built according to expected performances. The error bar is estimated to be $4.8 \%$ of the transmittance value. The total transmission was measured in all these configurations and found to be about $50 \%$ for tilted beams at $\left[0-10^{\circ}\right]$ angles, $40 \%$ for beams at $\left[10^{\circ}-20^{\circ}\right]$ angles and $30 \%$ for beams at $\left[20^{\circ}-30^{\circ}\right]$ angles.

This method is now well established, and ready to be used for the flight model.

\subsection{Wavelength dependence}

The efficiency varies with the wavelength of the received light. The bottom of Fig. 6 shows the filter and photocathode dependence. If the phenomenon to be observed is Nitrogen fluorescence (showers), then the spectrum is shown in Fig. 6 top for atmospheric pressure. It is clear that due to the finite number of lines, the efficiency will be very specific. If, furthermore, at low-pressure the strong $337 \mathrm{~nm}$ line becomes very weak and the $391 \mathrm{~nm}$ grows to double its size, it means that the efficiency when detecting showers will depend on the shower altitude. A similar effect exists for the lenses material, more specifically PMMA.

The JEM-EUSO flight optics lenses will be manufactured in Japan by Ikegami Mold, Inc. and will be inspected following manufacturing. Measurements of the surface roughness of Fresnel facets and diffractive gratings at the center, middle and edge of the lenses will be made. We expect the surface 


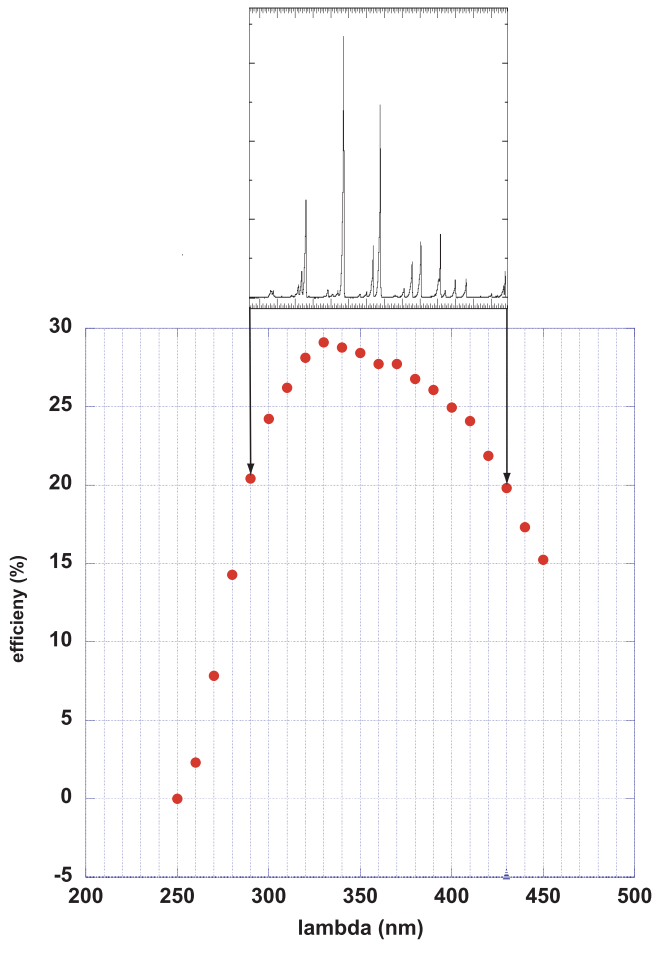

Fig. 6 Bottom: Wavelength dependence of efficiency due to photocathode and BG3 filter. Top: spectrum of nitrogen fluorescence at atmospheric pressure.

roughness between 10-20 $\mathrm{nm}$ rms. The results of these measurements will be compared to manufacturing errors (tolerances) specified for the optics to meet JEM-EUSO requirements as determined by optical simulations. These simulations should establish limits on surface roughness (or errors at high spatial frequency), on radial and tangential slope errors (at lower spatial frequencies) and on plunge cut depth errors in the blazed grating. Following inspection each lens will be mounted in its flight frame, aligned and an optical test on each lens will be performed. After integration into the frames and testing, each lens will undergo vibro-acoustic testing and following the vibration test another optical test will be performed to ensure the optics are still aligned and can survive the launch environment. Then each lens will be cleaned and installed in a flightlike metering structure for optical performance testing of the complete Optics Module (OM). These tests will be conducted in a space-like environment at MSFC's X-Ray and Cryogenic Facility (XRCF). A large-diameter collimated light source will be installed in the XRCF, permitting test and verification and full-aperture optical testing of the OM. The tests will include measurements of throughput and spot size over the full range of operational temperatures. In addition, the OM assembly will be tested at survival temperature ranges to ensure the lens frames can maintain optical alignment. For testing of the flight optics three large collimators in the United States have been identified for JEM-EUSO ambient and thermal testing. These included: a) the $2 \mathrm{~m}$ spher- 
ical primary (radius of curvature $\mathrm{RoC}=24.83 \mathrm{~m}$ ) on Redstone Arsenal; b) the $2.54 \mathrm{~m}$ parabolic primary $(\mathrm{RoC}=15.24 \mathrm{~m})$ at Wright-Patterson Air Force Base (AFB); c) the $3 \mathrm{~m}$ spherical primary $(\mathrm{RoC}=12.19 \mathrm{~m})$ at Arnold AFB. The first two candidate primaries are locked into expensive facilities and cannot be relocated. The $3 \mathrm{~m} \mathrm{f} / 2$ primary at Arnold was part of a solar simulator facility but has since been decoupled from that role and is in storage. It is potentially available for relocation to MSFC.

When considering the testing to be done on the flight optics the experience gained previously on BBM testing will prove useful. The type measurements recommended include the following: a) Effective Focal Length (EFL) via lateral magnification b) Back Image Distance; c) Axial Color (over EUSO wavelengths); d) F-number; e) Axial Transmission (over EUSO wavelengths); f) PSF tests (over EUSO wavelengths); g) Image Resolution (within EUSO limits); h) Relative Illumination Falloff (within allowable chamber boundaries); i) Field Curvature (over EUSO wavelengths, \& allowable chamber boundaries); j) Stray Light Effects (veiling glare). Details of these various tests need to be fleshed out and here the work done on the BBM will prove helpful not only for what was accomplished but also for problems encountered. Not all the tests listed above were done on BBM due to equipment, time, \& budget limitations. The transmission tests both in acquiring incident \& transmitted power measurements were particularly challenging. PSF imagery was acquired in both analog \& digital fashion. The former images were rather messy. Some oddities were also experienced such as multiple axial image locations, and low level fluorescence in the UV fiber optic source cabling. Quantitative stray light measurements were not made but its presence was qualitatively evident to the eyeball. Surface roughness measurements were made at sample locations using a replication technique. But these measurements were not incorporated into a stray light model.

\section{On-board Calibration}

\subsection{Relative in-flight calibration}

Gains and efficiencies may change or drift during the mission on-board the ISS. Gains are achieved by the electron multiplication at the dynodes. If this electronic current becomes too high, damage to the dynodes (especially in the last dynodes). Hamamatsu recommends keeping the combined anode current (of all 64 anodes) less than or equal to $100 \mu \mathrm{A}$. On JEM-EUSO, where the normal gain of $1.0 \times 10^{6}$, the background from stars and air glow creates a signal of about $1.2 \mathrm{MHz}$ of spe/pixel, or a $77 \mathrm{MHz}$ spe frequency per PMT which corresponds to $12 \mu \mathrm{A}$. So we have a usable signal range of only $90 \mu \mathrm{A}$ above the background. As a safeguard, the PMTs are protected by a switching system, where only the voltage on the cathode is reduced, but in a very short time $(<2 \mu \mathrm{s})$. This reduction, instead of interruption, allows also to continue measuring the intensity of bright events, for the following reason: the voltage 
on all other dynodes remains the same. The technique reduces the collection efficiency, by a factor of about 100 if a predetermined threshold corresponding to $100 \mu \mathrm{A}$ is reached in one $\mathrm{EC}$ (which means that $100 \mu \mathrm{A}$ at $-900 \mathrm{~V}$ will be seen as $1 \mu \mathrm{A}$ at $-750 \mathrm{~V}$. If the light is still so important that at $-750 \mathrm{~V}$ we have again more than $100 \mu \mathrm{A}$, then the applied voltage to the cathodes is $0 \mathrm{~V}$, for a total collection efficiency reduction of $10^{4}$. The result is that the gain of the tube is unchanged, only the number of electrons arriving at the first dynode is changed. Thus the tube always works in spe mode.

Finally, a small gain change has a negligible effect on the experiment because the discriminator in the ASIC is set in the minimum shown in Fig. 1, where the slope is horizontal. If, however, the gain has a substantial change, then it must be corrected by changing the HV, which will also change the efficiency.

Under normal conditions the quantum efficiency does not change: the cathode thickness is so small that its hadroninic cross-section is very weak. However, the transparency of the glass on which the photocathode is deposited and the UV filter (also composed of glass) can be reduced. This could happen due to contamination prior to launch or volatiles re-condensing on the filters during the space mission. Yet another cause of efficiency loss is radiation damage. On the ISS, this latter effect should be small because the radiation dose in low earth orbit is relatively small. The lens transparency can be also reduced by contamination or re-condensation and radiation effects. Due to these factors, the efficiencies have to be regularly measured during the mission. It is not possible to re-calibrate the focal surface during the mission, even with the help of NIST photodiodes, because these diodes can also suffer from contamination and re-condensation. See also next section for a detailed description of in-flight calibration. The GLS, as described later, could provide an independent cross check over the entire mission.

\subsection{Method of the on-board calibration}

As described in the previous section, the JEM-EUSO focal surface detector will be accurately calibrated against a standard reference before launch. Repeating the same calibration procedure on orbit is not possible. However, the degradation of the focal surface detectors and the lenses with time will be monitored on-board with LEDs. The light output of LEDs is known to be temperature dependent. To monitor changes in the light output of the LEDs each LED will be controlled by a NIST photodiode, mounted in a small integrating sphere. The photodiode is not polarized and is very stable against radiation. Its temperature dependence is well known and quite small.

In addition, the GLS provides external calibration sources [11]. In clear sky conditions, the attenuation of the flasher and laser signals is caused only by Rayleigh scattering and distance. Corrections can be calculated for both. The intrinsic luminosity of the flashers and the power output of the lasers are calibrated and monitored during the mission. Furthermore, the possibility of 
using the moon (an external and well-known light source) will be investigated for JEM-EUSO [10].

Since the on-board resources are limited, the measurement of the light collection efficiency will be restricted to measure the relative change, but not the absolute scale. However, all gain changes will be measured absolutely.

(a) Detector calibration

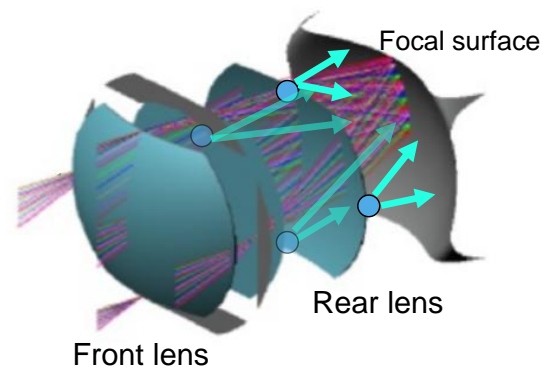

(b) Optics (+detector) calibration

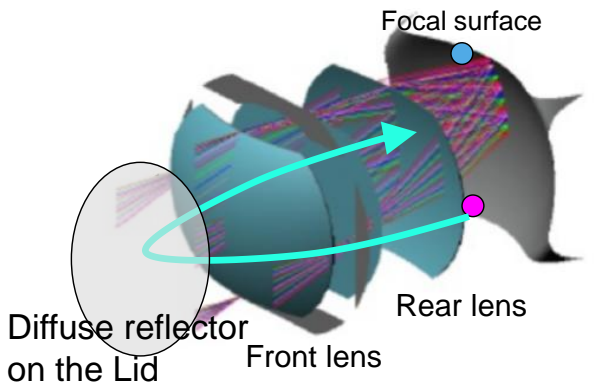

Fig. 7 On-board calibration system. Diffused UV light sources made of integrating spheres will be set at the position shown in the panels (a) and (b), and the time variation of the efficiency of the optics and the detector will be monitored. (a) Several light sources will be set along the edge of the rear lens to illuminate the focal surface directly. The relative change of the detector efficiency will be taken. (b) The same light sources are placed along the edge of the focal surface to illuminate the rear lens. The light is reflected back at the diffuse surface on the lid and is detected by the focal surface detector. Here, convolution of the efficiency of the optics and that of the detector will be obtained.

\subsection{On-board calibration device}

The light source consists of a small integrating sphere of 1-inch diameter equipped with one or more UV LEDs in 290 - $430 \mathrm{~nm}$ and a calibrated photodiode to monitor the variation of the light intensity (Fig. 8). The candidate integrating sphere for JEM-EUSO is 3P-GPS-010-SL by Labsphere [12], which has a internal diameter of 1 inch and Spectralon as diffusing material. Several identical light sources will be located behind the third lens and illuminate the entire focal surface (Fig. 7, left). The intensity will be set at single photoelectron level by stable DC light, and the photon detection efficiency of the system will be obtained in relative way, while the gain of MAPMT will be measured absolutely. If a large change of gain is found, the threshold level will be adjusted. Other several light sources will be set along the edge of the focal surface to illuminate the third lens (Fig. 7, right). The light passes through the lenses and is reflected back at the diffuse surface on the lid. Sand-blasted aluminum is the best candidate for the lid surface as it has a high resistance 

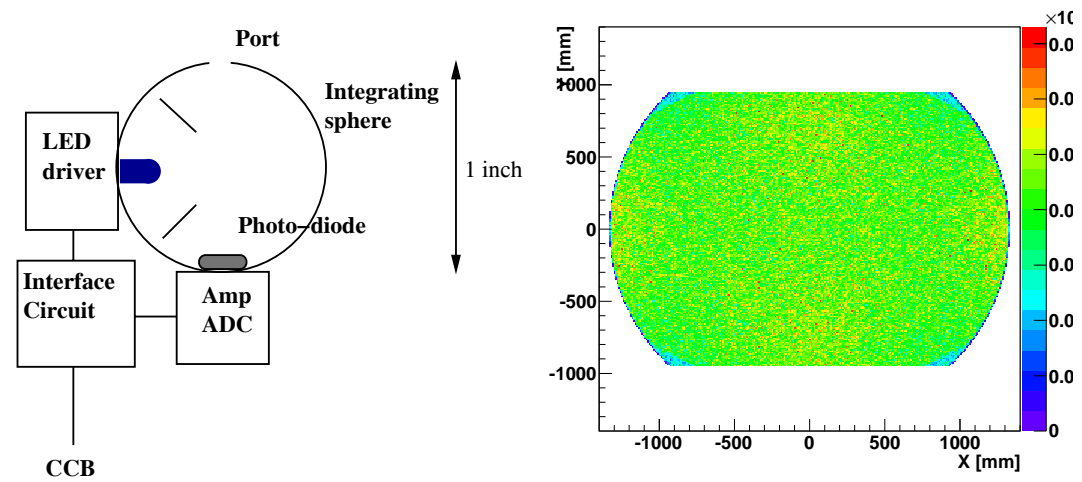

Fig. 8 Left: Schematic view of the light source. The light source consists of one or more UV LEDs, monitor photo-diode, LED driver electronics, readout electronics and interface circuit to a cluster control board (CCB). Right: The light intensity distribution on the focal surface. The shadow color scale shows the photon detection probability in $1 \mathrm{~cm}^{2}$ area when the light source emit one photon.

to the harsh space environment. The time variation of the performance of the optics and the detector will be obtained at the same time by this measurement. Therefore, after subtracting the degradation of the detector itself, the change in the optics throughput will be obtained.

\subsection{Expected performance}

Direct illumination: The source emits photons from a pin hole to the direction following the Lambertian distribution. The maximum emitting angle will be 60 degrees from the optical axis of the light source. The size of the light source (integrating sphere) was neglected in the calculation. The light sources are put at the edge of the rear lens on $x=0$ or $y=0$ line. The optical axis of the source is inclined from the optical axis of the telescope by 50 degrees. Fig. 8 (right) shows the illumination pattern on the focal surface when 4 light sources deployed on the third lens. The pattern is very uniform and needs to be very stable during operation. If one of the four light sources fails the pattern at the focal surface is not uniform anymore, but still acceptable for the calibration. Therefore, 4 sources provides a redundancy of the system.

Illumination through the optics: For the measurement of the optics transmittance, light sources are placed at the edge of the focal surface. The emission angle is narrowed down to \pm 10 degree, because the lid is assumed to be totally diffusive (Lambertian reflector). This reflection pattern has been confirmed by quick-look measurement of sand-blasted aluminum. The inclination angle from the optical axis of the telescope is set to 10 degrees. The reflectivity of the lid is assumed to be $50 \%$. 


\section{Absolute In-Flight Calibration}

The calibration work during orbit will be performed in three steps. First, the counting rate of each pixel will be monitored during the ultra-high energy cosmic ray (UHECR) observation for a health check. Based on this information, dead pixels will be disabled online or ignored in the offline analysis. In the present design, a 'slow mode' is defined where the count rates are monitored every 3.5 seconds. The slow mode data can be used for the calibration to derive average counting rate in each orbit. Second, more detailed calibration work will be performed every orbit, before or after the dark sky observations. In this stage, the efficiencies of each pixel of the focal surface detector with the light sources will be measured on the third lens at a few different light intensities allowing the efficiency to be measured in single photon counting mode and for strong light. The signal in darkness will also be measured. Third, more detailed calibration data will be taken every month. The absolute gain of each pixel will be determined by photon counting, making use of the threshold scan method (i.e. taking 'S-curve'). The transmittance of the optics will be also examined at this stage. The average rate of the calibration data will be a few kbps, which is well below the allocated rate for calibration data.

Two methods are foreseen: moon illumination, and calibrated man-made light from outside (GLS)

\subsection{Moon calibration}

The moon flux is well known. In order to have the smallest error on its reflection on clouds, one would limit ourselves to high altitude $(10 \mathrm{~km})$ thick enough for the optical thickness to be greater than unity (so that reflected light does not depend too much on incident or emergent angles and the albedo would be around $80 \%$ above ice or snow). This would be monitored by the atmospheric monitoring system (AMS), and predictions tell there are such clouds on about $7 \%$ of the time on the ISS path. The spectral irradiance of the moon at the ISS altitude is well known. The light intensity in that case would be about 400 pe $\cos \theta_{0} R /\left(\mathrm{GTU} \cdot\right.$ pixel) for a full moon, and $25 \cos \theta_{0} R$ pe for a half moon ( $\theta_{0}$ being the zenith angle of the moon and $R$ being the albedo of the cloud). This is in agreement with the ASIC front-end electronics possibilities. At half moon, it would take 10000 GTUs for half moon at zenith and angle of $60^{\circ}$. The ISS moves only $200 \mathrm{~m}$ in that time, less than the projected size of a pixel on ground. So, this method is possible and the accuracy would be of the order of $20 \%$ or less.

\subsection{Global Light System (GLS)}

In addition to extensive air showers (EAS) and transient luminous events, JEM-EUSO will also record optical calibration signals generated by a global 
network of calibrated UV light sources, the GLS [13] (Fig. 9). UV light from GLS xenon flashlamps will appear as optical point sources located deep in the atmosphere. In addition, light scattered out of the UV laser beams will create the appearance of an EAS track. The wavelengths of these sources will overlap major lines in the fluorescence spectrum of electrons in air (Fig. 9, right panel). The technique draws on the successful experience of ground based fluorescence detectors that used flashlamps [14] and lasers [15] in various configurations. Their data demonstrated [16-18] that lasers observed from the side as 'test beams' produce a luminosity that is comparable to the high energy EASs. Extrapolations from the single laser frequency to the broadband emission of the shower signal is doable. The lasers in the GLS units will produce tracks in the JEM-EUSO observatory. The laser tracks will have optical similarities to the tracks produced by $100 \mathrm{EeV}$ EASs.

Unlike cosmic EAS events which are essentially random, the properties of GLS flashes and laser shots can be programmed in advance and measured independently at their source. The properties of the GLS signals include the absolute time, calibrated intrinsic luminosity for the flashers and calibrated beam energy and direction for the lasers. These independent measurements can then be compared, event by event, to the JEM-EUSO measurements of these GLS signals to monitor and validate key parameters of the detector and the data analysis chain. Parameters include trigger efficiency, pointing accuracy, and accuracy of intrinsic luminosity reconstruction.
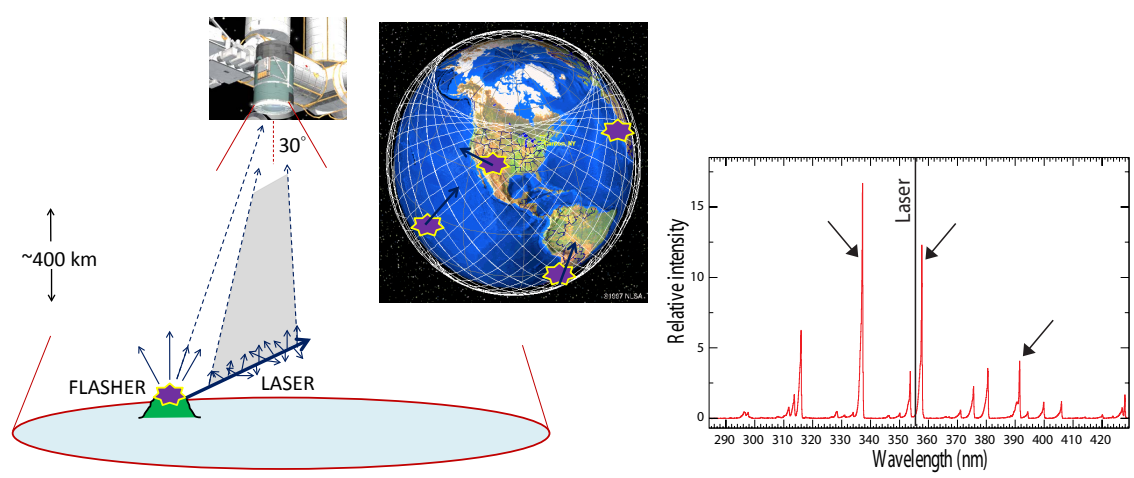

Fig. 9 Left: The signals from the Global Light System of ground-based calibrated xenon flashlamps and lasers will be measured by JEM-EUSO to monitor its performance during the mission. Right: This is the nitrogen fluorescence spectrum (Spectrum shown is from reference [19]). The wavelengths of the narrow band filters uses with the xenon flash lamps are indicated by arrows. In addition to these narrow filters, the flash lamps will use a broadband SCHOTT BG3 filter. Also indicated by the black line is the wavelength of the laser $(355 \mathrm{~nm})$. 


\subsubsection{Configuration and Applications}

The GLS will include 12 ground-based stations located at remote sites around the globe at altitudes between 3 and $4.5 \mathrm{~km}$. All sites will include calibrated Xenon flashlamps. Six of the twelve sites will include steerable laser systems. In addition, a portable system with a laser and flashlamps will be deployed monthly over the open oceans by an aircraft. The ground based systems will be operated and programmed remotely.

The number of passes JEM-EUSO will make over GLS stations under favorable atmospheric conditions will roughly equal the number of EASs that JEM-EUSO will record above $5 \times 10^{19} \mathrm{eV}$. JEM-EUSO will pass over a ground station at night with favorable viewing conditions about every 48 hours on average. This estimate of times between measurement opportunities was obtained by a model that required the sun be at least $18 \mathrm{deg}$ below the horizon, the illumination of the moon be less than $50 \%$ and assumed the chance of favorable conditions to be $33 \%$. This is approximately the fraction of time that the sky will be clear as viewed from the ground. The average crossing time of the JEM-EUSO footprint over a GLS station is about 60 seconds. The combined stations will alternate laser shots and xenon flashes to provide a set of measurements across the JEM-EUSO field of view. For each pass, the atmospheric monitoring system (AMS) on board the JEM-EUSO will acquire IR camera images and a LIDAR scan aimed at the location of the GLS site[5].

There will occasionally be very clear conditions when the measured total optical depth is not significantly greater than the molecular optical depth. At these times the intrinsic luminosity of the flashers can be predicted by knowing the range to the GLS and by accounting for Raleigh scattering in the intervening atmosphere. In such cases, the GLS becomes a reference calibration source for the efficiency of JEM-EUSO. When measurements of the flasher show that atmospheric scattering is dominated by Raleigh scattering, the light scattered from the laser beam along its length can be calculated. Since the energy in the laser beam is known, the intrinsic luminosity of light scattered from the beam is also known and can be compared to the intrinsic luminosity reconstructed from measurements made by JEM-EUSO.

GLS lasers will also be programmed to generate an artificial full sky map of potential cosmic accelerators by firing laser shots at astronomical objects of interest such as Cen-A, Virgo, and the galactic center. A sky map of laser track directions as reconstructed by JEM-EUSO will be accumulated over the mission to test the absolute EAS pointing accuracy reconstruction using the JEM-EUSO instrument, including the correct generation and transfer of absolute time stamps through the data acquisition and analysis chains.

GLS sites will be selected to represent the variety of terrestrial backgrounds over which JEM-EUSO is expected to measure EASs. Selection criteria for sites include low light backgrounds, an altitude above the typical planetary aerosol boundary layer for that site, physical and legal access, and possibility for communications link. Since oceans represent the bulk of the dark regions, 
sites on isolated mountainous islands are especially desirable, as are sites with existing scientific installations that include atmospheric monitoring.

\subsubsection{Xenon Flashlamp}

Since JEM-EUSO will look down on the atmosphere, intrinsic luminosity reconstruction can be monitored directly with flashlamps [20]. All 12 GLS stations will include 4 individual flashlamps (Hamamatsu L6604). The L6604 model features a highly stable output with $<3 \%$ shot-to-shot stability, a stable lifetime of more than $10^{7}$ pulses and $<3 \%$ degradation over the lifetime of the mission [21]. The light pattern from each flash is smoothly distributed over a wide field of view. These key performance parameters have been verified in laboratory tests. The flash duration from the Hamamatsu L6604 lamp has been stretched to achieve widths 20 microseconds (Fig. 10) while maintaining consistent flash output with deviations less than $5 \%$. Three flashlamps will be filtered to match the primary lines indicated in Fig. 9, and the fourth will use a broad band (SCHOTT BG3) transmission filter identical to the filter planned for the JEM-EUSO detector. A photo-diode will measure the relative output for each flash. The flashlamp point images can also be used to monitor the focus of the telescope throughout the mission and to test the accuracy of its pointing determination.

GLS: Filter BG3
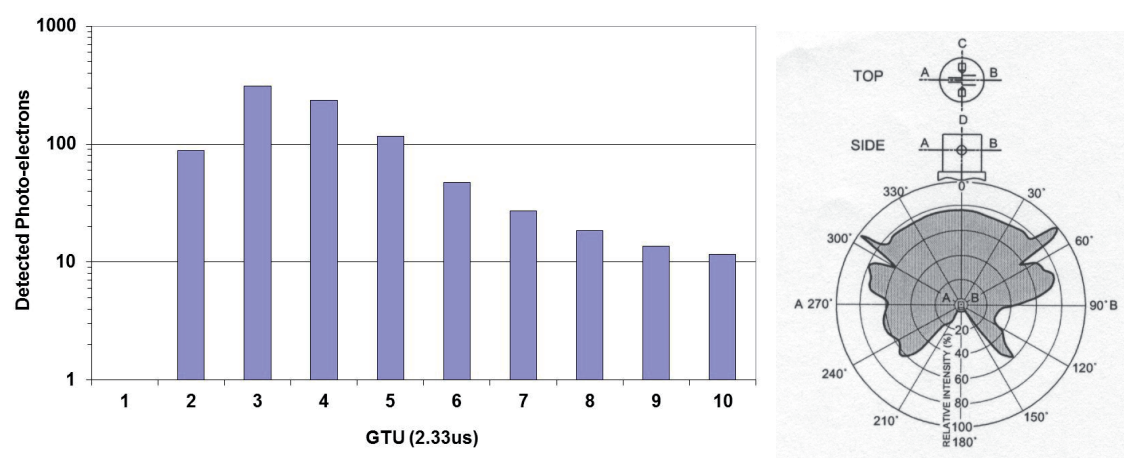

Fig. 10 Left: Estimated number of photo-electrons detected by the JEM-EUSO telescope from a stretched UV-flasher pulse. The long duration pulse has been achieved by customizing the HV output. The output variation remains with the requirements needed for mission. Right: Intensity distribution of the L6604 UV-flasher output.

\subsubsection{Laser Systems}

The design and performance parameters draws on that of two laser facilities that have been in operation at the Pierre Auger Observatory since 2004 and 


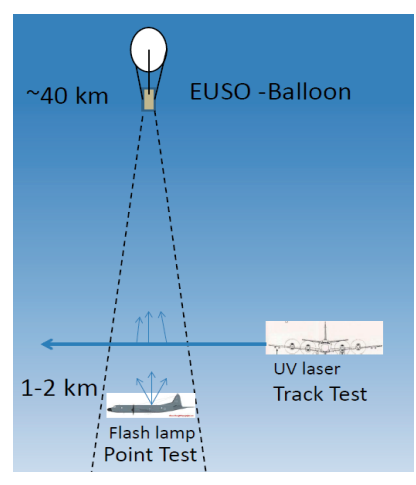

Fig. 11 To test the EUSO-Balloon prototype detector an aircraft will fly under and next to the detector field of view with portable flasher and laser sys-

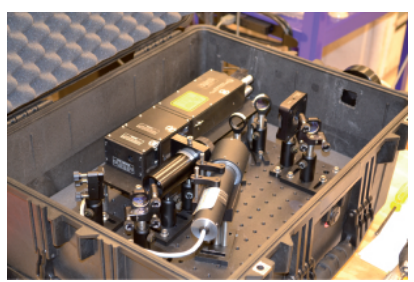
tems (left panel). A portable laser system is being developed for the EUSO-Balloon tests. The insert shows the control computer and GPS timing unit (right panel).

2009. The laser will be a frequency tripled YAG with an output wavelength of $355 \mathrm{~nm}$. The relative energy of each shot will be measured by a pyroelectric energy monitor probe. The net polarization of the beam will be randomized so that the atmosphere scatters equal amounts of light about the direction of the beam axis. For calibration of the monitor energy probe, the beam steering system directs the beam downward to a calibration probe that will measure the absolute beam energy downstream of all optics. To facilitate separation of EAS and laser data collected by JEM-EUSO, the laser will be triggered at precise times using a custom GPS timing module [22]. The time, direction, and energy of each shot will be recorded locally.

\subsubsection{Aircraft Systems}

A portable GLS system with flashers and a laser pointed horizontally will be installed in a P3B airplane managed by the NASA Airborne Science Program (ASP). The airplane will be deployed monthly for under-flights of the ISS at night. The P3B will fly out $500 \mathrm{~km}$ from NASA Wallops Flight Facility on the east coast of the U.S. to rendezvous with the ISS over the north Atlantic ocean for a single under-flight.

\subsubsection{Testing EUSO-Balloon}

A prototype airborne GLS system will be deployed in an aircraft to support the suborbital EUSO-Balloon mission [23-25] (Fig. 11, left panel). This 2014 mission will test the JEM-EUSO concept and technique as well as the operation of its key components. It will also measure the UV background below $40 \mathrm{~km}$. To study the sensitivity to EASs, EUSO-Balloon will measure flashes and tracks from the airborne GLS system. The laser system under development for this test is shown in Fig. 11, right panel. 


\section{Conclusion}

The calibration scheme of the JEM-EUSO instrument will be organized in three major steps:

\section{At the laboratory on ground:}

An absolute efficiency measurement must be completed on the ground before launch, since this will not be possible on-board. The gain and the efficiency of each individual pixel in each photomultiplier will be absolutely determined. These characteristics need to be determined with an accuracy better than $5 \%$. As the light to be detected is very weak, the photomultiplier tubes (PMTs) will work in a 'single photoelectron' (SPE) counting mode.

\section{On the launch pad:}

By using the calibration devices installed in the instrument, the absolute gains will be verified and the relative efficiencies will be measured with an statistical accuracy of better than $1 \%$ for each individual pixel. These efficiencies will be normalized to the absolute values as determined before flight.

\section{During flight:}

By an on-board device: The same procedures as in step two will be performed periodically to monitor and verify the gains as well as to compare, pixel by pixel, the SPE spectra. In this way, drifts in the gain can be corrected by adjusting the high voltages. Recognized drifts in the efficiency however, need to be handled differently as they cannot be corrected. If the drift is not more than (5\%), it will be accounted for during the reconstruction of the measured events. If the drift is larger, the values of the calibration tables will be changed accordingly. The on-board calibration devices will allow us to determine the source of such drifts. In particular it can distinguish the origins of either a malfunction of the focal surface detector or of the optical system.

By external calibration devices: (i) Reflected Moon light will be used for a cross-check of the on-board calibration, where a precision of $20 \%$ can be reached. (ii) The use of calibration with a Global Light Source (GLS) system on ground illuminating the instrument in space can reach a precision of better than $15-20 \%$. Both methods (i) and (ii) will be studied in detail, as a redundant calibration scheme during the flight time of the instrument is required.

Acknowledgements This work is partially supported by Helmholtz Alliance for Astroparticle Physics, Germany, and by NASA grants NNX13AH55G, NNX13AH53G.

\section{References}

1. Adams Jr., J.H. et al. [JEM-EUSO Collaboration], An evaluation of the exposure in nadir observation of the JEM-EUSO mission, Astroparticle Physics, 44, 76-90 (2013) 
2. Adams Jr., J.H. et al. [JEM-EUSO Collaboration] The JEM-EUSO mission, This volume.

3. Adams Jr., J.H. et al. [JEM-EUSO Collaboration] Ultra High Energy Cosmic Rays studies with the JEM-EUSO mission, This volume.

4. Adams Jr., J.H. et al. [JEM-EUSO Collaboration] The JEM-EUSO instrument, This volume.

5. Adams Jr., J.H. et al. [JEM-EUSO Collaboration] The Atmospheric Monitoring System of the JEM-EUSO instrument, This volume.

6. Adams Jr., J.H. et al. [JEM-EUSO Collaboration] The Photodetector Module of the JEM-EUSO mission, This volume.

7. Adams Jr., J.H. et al. [JEM-EUSO Collaboration] Performances of JEM-EUSO: energy and $\mathrm{X}_{\max }$ reconstruction, This volume.

8. Adams Jr., J.H. et al. [JEM-EUSO Collaboration] Performances of JEM-EUSO: angular reconstruction, This volume.

9. Blaksley, C. et al. [JEM-EUSO Collaboration], Proceedings 33rd International Cosmic Ray Conference, Rio de Janeiro, 2013, paper 628.

10. Sakaki, N. et al. [JEM-EUSO Collaboration], Proceedings 33rd International Cosmic Ray Conference, Rio de Janeiro, 2013, paper 546.

11. Wiencke, L. et al. [JEM-EUSO Collaboration], Proceedings 33rd International Cosmic Ray Conference, Rio de Janeiro, 2013, paper 718 .

12. General purpose integrating sphere, http://www.labsphere.com/products/spheres-andcomponents/general-purpose-spheres/default.aspx, Labsphere Inc.

13. Adams Jr., J.H. et al. [JEM-EUSO Collaboration], Advances in Space Research, 53, $1506(2014)$

14. Wiencke, L. et al., [HiRes Collaboration], Nucl. Instrum. Meth. A, 428, 593 (1999).

15. Fick, B., Malek, M., Matthews, J., Meyhandan, R., Mostafa, M., Roberts, M., Sommers, P., and Wiencke, L., JINST, 1, p11003 (2006) [arXiv:astro-ph/0507334].

16. Bird, D.J. et al., Ap.J., 441, 144 (1995) [arXiv:astro-ph/9410067].

17. Cannon, C., Pedersen, L., Riehle, R., Thomas, J. and Wiencke, L. [for the HiRes Collaboration], in Proceeding of the 28th International Cosmic Ray Conference (Tsukuba), 477-480 (2003).

18. Abreu, P. et al. [Pierre Auger Collaboration], JINST, 8, p04009 (2013). [arXiv:1303.5576]

19. Ave, M. et al. [AIRFLY Collaboration], Astropart. Phys., 28, 41 (2007).

20. Gorodetzky, P., Sakaki, N., Christl, M. [JEM-EUSO Collaboration] Proceedings of the 32th International Cosmic Ray Conference (Beijing), 3, 36-40 (2011) [arXiv:1204.5065v1 [astro-ph.IM]].

21. Xenon Flash Lamps, Hamamatsu Photonics K.K, http://www.hamamatsu.com

22. Smith, J.D., Thomas, J.R., Thomas, S.B. and Wiencke, L.R., Proceedings 30th International Cosmic Ray Conference (Mérida), 5, 997-1000 (2008) (US patent 7,975,160).

23. http://euso-balloon.lal.in2p3.fr/

24. von Ballmoos, P. et al. [JEM-EUSO Collaboration], Proceedings 33rd International Cosmic Ray Conference (Rio de Janeiro), (2013), paper 1171.

25. Adams Jr., J.H. et al. [JEM-EUSO Collaboration] The EUSO-Balloon pathfinder, This volume. 